\title{
Sex Bias in Preclinical Studies: The Case of Gut Microbiota on Alzheimer's Disease
}

\author{
M Pilar Vinardell* \\ Department of Biochemistry and Physiology, University of Barcelona, Spain
}

*Corresponding author: Department of Biochemistry and Physiology, Faculty of Pharmacy and Food Sciences, University of Barcelona, av. Joan XXIII 27-31, Barcelona 08028, Spain.
Received Date: July 15, 2021

Published Date: August 03, 2021

\begin{abstract}
Alzheimer's disease (AD) is an irreversible and progressive neurodegenerative disorder characterized by a loss of memory, language, and cognitive ability. It is the most common form of dementia in older adults and represents a major public health problem. Changes in the gut microbiota have been investigated in the development of $\mathrm{AD}$, with studies on this from the last five years using different models of AD in laboratory animals, mainly transgenic mice. Despite the fact that the majority of people diagnosed with AD are women, preclinical studies are still being carried out with male animals. In this review, we analyze the most recent studies on the gut microbiota in AD, recording the methodology used and the presence of sex bias. From the 35 studies selected, $65 \%$ used male animals and only $20 \%$ used both sexes.
\end{abstract}

Keywords: Alzheimer's disease; Animal model; Sex bias; Gut microbiota; Gut-brain axis

\section{Introduction}

Alzheimer's disease (AD) is an irreversible and progressive neurodegenerative disorder characterized by a loss of memory, language, and cognitive ability. It is the most common form of dementia in older adults and is a major public health problem. $\mathrm{AD}$ is a progressive illness that requires adequate interventions to prevent or reduce disease progression. $\mathrm{AD}$ is a complex multifactorial disease that appears to be unique to humans. The age at onset, rate of progression and the development of pathology are highly variable between patients. $\mathrm{AD}$ is characterized by a pathological accumulation of amyloid $\beta(A \beta)$ in the extracellular plaques of the brain parenchyma and vasculature as well as by abnormally phosphorylated tau that accumulates in the neurons to form neurofibrillary tangles. The pathological aggregation of $\mathrm{A} \beta$ and phosphorylated tau occurs in a sequential process. Small numbers of monomers first aggregate into oligomers into neurons, which then continue to aggregate into the fibrils observed in amyloid plaques and neurofibrillary tangles [1]. Amyloid plaques primarily consist of aggregated $A \beta$. There are different $A \beta$ species such as $A \beta 1-40, A \beta 1-42, A \beta 1-38$, and $A \beta 1-43$, among others. The presence and amount of these different $A \beta$ species are important because each species has a different rate of aggregation and forms different aggregated species, some of which are more toxic than the others.

The clinical diagnosis of AD is currently based on a decline in cognition and detection of amyloid $A \beta$ and tau levels in the cerebrospinal fluid. Diagnosis of AD is based on the postmortem neuropathological assessment of both amyloid plaques and tau pathology, which are not usually present both in AD animal models [2]. Amyloid deposition in the brain can lead to the activation of immune responses in the brain. $A \beta$ deposits activate the microglia, which are innate immune cells in the brain. This activation leads to an inflammatory response in the central nervous system (CNS), known as the amyloid cascade. The amyloid cascade hypothesis is the main hypothesis for the pathophysiology underlying $\mathrm{AD}$, with 
the amyloid deposition possibly beginning 10-20 years before the first symptoms of cognitive decline and AD diagnosis [3]. An increase in the permeability of the blood-brain barrier has been observed in AD. The human gut presents a diverse population of microorganisms, known as the gut microbiota. The most abundant in the adult human intestine are Firmicutes and Bacteroidetes [4] It is known that gut microbes may alter levels of neurotransmitterrelated metabolites, affecting gut-to-brain communication and brain functions. Recent studies suggest that the gut microbiome can play an important role in the progression of neurodegenerative diseases such as AD [5-7].

\section{Materials and Method}

\section{Microbiota and neurodegenerative disorders}

There is an axis between the microbiota, gut and brain (MGB axis) observed in many studies including neurodevelopmental disorders such as autism [8,9]. Different experiments suggest that the gut microbiota and probiotic agents can alter levels of circulating cytokines, which can have a significant effect on some brain functions. Alterations in the gut microbiota can modulate the peripheral and central nervous systems, resulting in altered brain function [10]. The specific role of the gut microbiota in modulating neuroimmune functions may have an important effect on the process of neurodegeneration, especially that associated with AD. The different components of the human gut microbiota, secrete amyloid proteins in the CNS, resulting in $A \beta$ accumulation and an increased risk of $\mathrm{AD}$ [11]. A healthy gut has a normal and stable commensal intestinal microbiota that provides the host with nutrition and energy by producing vitamins. Gut-related inflammation is essential in provoking endotoxemia, systemic inflammation, and neuroinflammation, which can contribute to neurodevelopmental disorders and the onset of psychiatric illnesses in later life. Previous studies have provided evidences of the effect of the gut microbiota on the CNS, as well as its neuroinflammatory role in neurodegenerative diseases like AD [12]. Pro-inflammatory cytokines secreted by the microglia and astrocytes induce continuous $A \beta$ deposition and create a positive feedback that exacerbates neurodegeneration. The chronic gut inflammation increases intestinal permeability, leading to the release of more pro-inflammatory factors in the CNS Thus, it is essential to conduct future studies on cell signaling and cytokine regulation in the suppression of neuroinflammation in $\mathrm{AD}$ [13]. A strategy on the treatment and prevention of AD may be restoring and remodeling the composition of the gut microbiota. The majority of the data on the role of the gut microbiota in $\mathrm{AD}$ are from animal studies. But unfortunately, most preclinical animal studies end up with unexpected failures during clinical transition due to unidentified reasons [14].

\section{Sex differences in Alzheimer and other diseases}

Women have a greater lifetime risk of developing AD because women live longer than men, but longevity is not the only responsible for $\mathrm{AD}$ development [15]. Greater female longevity is found among chimpanzees and most rat species, but not among mice and orangutans [16]. A study of the impact of sex on AD found that nearly two-thirds of all patients diagnosed with $\mathrm{AD}$ are women. Furthermore, $16 \%$ of women, but only $11 \%$ of men, live with dementia after 71 years of age. The lack of consideration about the effect of gender in studies on AD has difficulted faster advancement in the detection, treatment, and care of $\mathrm{AD}$ and greater attention to these differences should be considered [17]. In order to guarantee the inclusion of female participants as well as racial or ethnic minority participants in clinical research, the National Institutes of Health Revitalization Act of 1993 established legal requirements and guidelines [18]. However, underrepresentation of female participants relative to disease prevalence (known sex bias) still persists in many studies $[19,20]$. In 2015, new NIH policies were announced and require applicants to report the balance of male and female in studies with cells and animals in preclinical studies for all applications, except in some special cases where it is necessary to demonstrate the need for only one sex. The policy requires that sex should be considered as a biological variable and should be included into study designs, analyses, and reporting in animal studies, with limited exceptions. It further requires sex difference analyses to be performed in most studies, prescribing that researchers should collected data disaggregated by sex [21].

The higher rates of adverse drug events in women are the primary motivation for the inclusion of both male and female materials in all preclinical research. When female patients receive treatment based on the results of studies on male participants, adverse events may occur because of sex-specific differences in disease patterns, drug pharmacokinetics and clearance [22].

In a study on sex bias using a large amount of data from articles and records, the authors observed that there was substantial female underrepresentation in studies of different diseases and especially HIV/AIDS and chronic kidney diseases. When using studies as a measurement unit, there was little increase in female representation in the studies from 1993 to 2018; however, when using participants as a measurement unit, female representation had improved. Authors concluded that sex bias against female participants in clinical studies persists despite legal and policy initiatives to increase female representation. This sex bias against females is also present in previous preclinical studies with laboratory animals [23].

\section{Experimental models of Alzheimer disease}

Experimental models are essential to understand the pathogenesis of $\mathrm{AD}$ and to study the potential effects of novel treatments performing preclinical testing. To date, the majority of experimental models are based on transgenic mice that express human genes to induce the formation of amyloid plaques and neurofibrillary tangles. The first transgenic mouse model with developed in 1995 with a substantial amyloid plaque. Since then, new transgenic models have proliferated, each with a different phenotype of AD-associated pathology. There is an interesting review on the different animal models used to study AD. Most of the 
animal models used in $\mathrm{AD}$ research are transgenic mice. Wild-type mouse with the amyloid precursor protein APP (isoform 695) has 97\% sequence homology with human APP with mice and humans showing differences in 3 amino acids within the $A \beta$ sequence. The Tg2576 mice express human APP with the double Swedish mutation APPK670N/M671L, which results in the significant overexpression of APP. The APP23 mice also express APP with the double APPK670N/M671L mutation. Various transgenic mouse models based on APP/PS1 have been developed to study AD. The most extreme APP/PS1 mouse model that is widely used is the 5xFAD model which express different APP mutations. The expression of five human genes associated with familial AD (FAD) mutations results in intraneuronal $A \beta$ accumulation at 6 weeks, followed closely by plaque formation at 2 months. Another animal model to study $\mathrm{AD}$ is transgenic mice expressing tau, because wild-type mouse tau does not develop neurofibrillary tangles. The most commonly used models of $\mathrm{AD}$ express $4 \mathrm{R}$ tau with P301L or P301S mutations, which result in neurofibrillary tangles, neurodegeneration, atrophy and motor deficits. The necessity of these mutations for the development of neurofibrillary tangles is an obvious limitation of these transgenic mouse models, as these mutations are not associated with AD in humans and the mutated tau may affect its toxicity or interaction with $A \beta$ in a way that is not representative of what occurs in AD. Furthermore, the overexpression of mutated tau elicits significant motor deficits that are not seen in $\mathrm{AD}$ and interfere with cognitive testing [24]. Only a few transgenic rat models of $\mathrm{AD}$ have been developed, despite having certain advantages over transgenic mice. They are more similar to humans in their physiological, morphological and genetic characteristics, while their larger brain makes cerebrospinal fluid collection, electrophysiology and imaging easier. Moreover, they have a behavioral phenotype, allowing for more complex behavioral testing [25]. Other animal models are based on the injection of $A \beta$ directly into the brain [26]. Irrespective of the animal model used to study AD and its treatment, sex bias occurs in these studies, similar to others.

It is necessary to perform research to determine the extent of publication bias in the laboratory animals' experiments [27]. In this sense, systematized reviews can be used to put an experiment into perspective, to refine the experimental design, to reduce the number of unnecessary animal experiments, lowering the risk of bias in the conclusions [28-35].

\section{Literature search}

In this review, we will discuss sex bias in the latest studies that have used these animal models to investigate how changes in the microbiota affect AD. A careful literature search was performed to find publications on the effect of the microbiota in animal models of AD. An online search was conducted on PubMed for studies published in English in the last 5 years using the following search term: animal model and Alzheimer and microbiota. The inclusion criteria were: (1) experimental AD induced in rodents (i.e., rats or mice) and (2) studies published in English. The exclusion criteria were: (1) not an original paper (e.g., review, letter, opinion, etc.), (2) studies in humans, (3) invertebrate models, and (4) other models

\section{Results and Discussion}

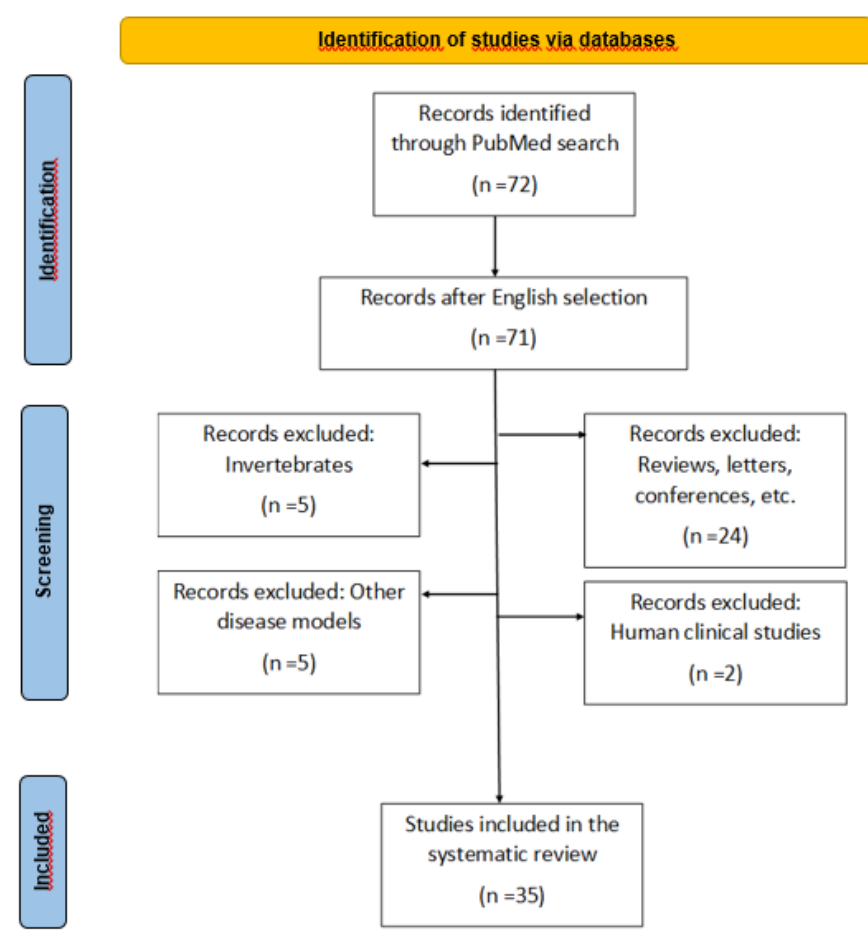

Figure 1: Flowchart showing how the papers entered the review based on the search. 
The search was limited to publications from the last five years because this corresponded to the period when all the papers related to the microbiota and AD were published. From a total of 72 articles related to $\mathrm{AD}$ and the microbiota, only 35 were reviewed based on the inclusion/exclusion criteria of the present review (Figure 1)

Table 1: Sex and animal species in different Alzheimer's models

\begin{tabular}{|c|c|c|c|}
\hline Animal & Sex & Alzheimer's model & Reference \\
\hline $\begin{array}{l}\text { Sprague Dawley } \\
\text { rats }\end{array}$ & Male & Amyloid- $\beta$ (25-35) for AD infused into the brain & 26 \\
\hline Mice & Male & $\begin{array}{c}\text { APP/PS1 transgenic mice (B6C3-Tg } \\
\text { (APPswe,PSEN1dE9)85Dbo/Mmjax; APP/PS1TG) }\end{array}$ & 29 \\
\hline $\begin{array}{l}\text { Wistar rats } \\
(180-220 \mathrm{~g}, 8 \\
\text { weeks of age })\end{array}$ & Male & Intrahippocampal injection of $A \beta 1-42$ & 30 \\
\hline Mice & Female & APP/PS1 heterozygous transgenic mice & 31 \\
\hline Mice (8-week-old) & Male & $\begin{array}{c}\text { Triple-transgenic mouse model of AD, B6;129-Psen1tm1Mpm Tg (APPSwe,tauP301L)1Lfa/J } \\
\text { (named 3xTg-AD) }\end{array}$ & 32 \\
\hline Mice & Male & $\begin{array}{c}\text { Triple-transgenic mouse model of AD, B6;129-Psen1tm1Mpm Tg (APPSwe,tauP301L)1Lfa/J } \\
\text { (named 3xTg-AD) }\end{array}$ & 33 \\
\hline Mice & Male & $\begin{array}{l}\text { AD triple-transgenic mice, B6;129-Psen1tm1Mpm Tg (amyloid precursor protein [APP]Swe, } \\
\text { tauP301L)1Lfa/J (named 3xTg-AD) and the wt B6129SF2 mice }\end{array}$ & 34 \\
\hline Mice & Both sexes & $\begin{array}{l}\text { Transgenic 5xFAD (APP K670N, M671L, I716V; } \\
\text { PS1 M146L, L286V }\end{array}$ & 35 \\
\hline Mice & Both sexes & $\begin{array}{l}\text { Transgenic Tg2576 mice, which harbor a human variant of APP termed the "Swedish" mutation } \\
\text { (APPswe), (Lys670/Asn and Met671/Leu), }\end{array}$ & 36 \\
\hline Mice & Male & (SAMP8) is a spontaneous animal model of overproduction of amyloid precursor protein (APP) & 37 \\
\hline Mice & Male & C57BL/ 6N transplanted with microbioma from Alzheimer patient & 38 \\
\hline Mice & Male & $\begin{array}{l}\text { Tg (APPswe, PSEN1dE9) 85Dbo are double transgenic mice and APP/PS1 heterozygous transgen- } \\
\text { ic mice }\end{array}$ & 39 \\
\hline Mice & Both sexes & APOE4 transgenic (E4FAD) & 40 \\
\hline Mice & Female & AppNL-G-F with pathogenic $A \beta$ are elevated & 41 \\
\hline Mice & No indicated & $\begin{array}{l}\text { Murine transgenic AD model, AD-like } \\
\text { pathology with amyloid and neurofibrillary tangles (ADLPAPT), }\end{array}$ & 42 \\
\hline Mice & Female & APPswe/PS1 $\Delta \mathrm{E} 9$ (PAP) transgenic & 43 \\
\hline Mice & Male & C57BL/6 local administration of $\mathrm{A} \beta$ peptide into the brain. & 44 \\
\hline Mice & Male & APP/PS1 transgenic & 45 \\
\hline Mice & Both sexes & APPSWE/PS1 1 E9 transgenic & 46 \\
\hline
\end{tabular}

[35-40].

All 35 publications were original articles reporting preclinical studies using mice or rats. Table 1 shows the different papers reviewed with indication of sex and animal species used and details about the different Alzheimer's models (Table 1). 


\begin{tabular}{|c|c|c|c|}
\hline Mice & Male & APPSWE/PS1 $\triangle \mathrm{E} 9$ transgenic & 47 \\
\hline Mice & Both sexes & $\mathrm{EFAD}(5 \mathrm{xFAD}+/-/ \mathrm{APOE}+/+)$ & 48 \\
\hline Rat & Male & Lyposaccharide rat model & 49 \\
\hline Mice & Male & SAMP8 and age-matched SAMR1 & 50 \\
\hline Wistar rats & Male & $\begin{array}{l}\text { A small hole was drilled on the skull and, via a Hamilton syringe, a single dose including } 10 \mu \mathrm{l} \\
\text { (containing } 10 \mu \mathrm{g} \text { ) of aggregated } \beta \text {-amyloid was injected into the lateral ventricles of the brain. } \\
\text { Two injections (containing a total of } 20 \mu \mathrm{g} \beta \text {-amyloid) were administered during two consecutive } \\
\text { days. }\end{array}$ & 51 \\
\hline Mice & Male & APP/PS1 double transgenic & 52 \\
\hline Mice & Male & APPswe/PS1dE9 double-transgenic & 53 \\
\hline Mice & Male & APPswe/PSEN1dE9 transgenic & 54 \\
\hline Mice & Both sexes & $\begin{array}{l}\text { P301L tau transgenic, expressing the longest human tau isoform (2N4R) with the FTDP-17 muta- } \\
\text { tion P301L in neurons }\end{array}$ & 55 \\
\hline Mice & Male & APPswe/PS 1dE9 (APP/PS1) transgenic (Tg) & 56 \\
\hline Mice & Female & $\begin{array}{l}3 \times \text { Tg-AD transgenic (TG) (RRID: } \\
\text { MMRRC 034830-JAX) harboring the APPSWE and TauP301L transgenes on a PS1M146V knockin } \\
\text { background (homozygous mutant APPSWE, PS1M146V, and TauP301L), }\end{array}$ & 57 \\
\hline Mice & Male & 5XFAD (Tg) express human APP and PSEN1 transgenes & 58 \\
\hline Mice & Both sexes & 5XFAD (Tg) express human APP and PSEN1 transgenes & 59 \\
\hline Mice & Male & 5XFAD (Tg) express human APP and PSEN1 transgenes & 60 \\
\hline Mice & Male & APP/PS1 transgenic & 61 \\
\hline Mice & Male & APPswe/PS1_E9 (PAP) transgenic & 62 \\
\hline
\end{tabular}

The majority of the studies were performed in mice, with only $4(11 \%)$ using rats. Among these studies, 66\% used only male animals, $11 \%$ used female animals and $20 \%$ assessed animals from both sexes (Figure 2). Only one study (3\%) did not indicate the sex of the animals employed (Figure 2) [41-62].

The high quantity of studies using only male animals is contrary to the upper incidence of AD in women [17]. There was a review of sex bias in research using non-human mammals in 10 different biological fields, where authors found male bias in 8 disciplines. Neuroscience was the one with most sex bias, with 5.5-time higher studies with only males than females [63]. These results were also observed within the period 2010-2014 [64] and similarly, in our results of the last five years. We found that there are only few comments in the studies discussing the sex of the animals used. One study defends the use of only female animals based on recent evidence indicating that women are at a greater risk of developing AD [57]. Another study mentioned that only female animals were included to avoid gender-specific differences related to the appearance of amyloid deposition during aging [31]. A recent study on the use of probiotics justified the use of only female animals [41] because an earlier work had demonstrated similar $A \beta$ deposition and gliosis across the sexes, but a more robust behavioral phenotype in females [65]. Therefore, authors elected to use female mice in the initial study but recognized that future work should 
compare probiotic effects in both male and female mice [41]. The fourth study using only female animals did not indicate the reason for this decision [43].

The studies using both sexes demonstrated differences between the genders, with the $A \beta$ deposition occurring earlier in female than in male animals [48]. However, Wang et al., in 2019 did not observe any sex-dependent changes in the composition of the gut microbiota and brain M1/Th1 cell abundance59, which are in contrast to the findings of Dodiya et al. in 2019 that showed sex-dependent changes in microglial numbers and $A \beta$ deposition during antibiotic treatment [66]. The authors speculated that this discrepancy might have resulted from the different experimental settings, such as the different mouse models used (APP/PS1-21 vs. $5 \times F A D)$, the varying ages of the mice receiving antibiotic treatment and the readout parameters.

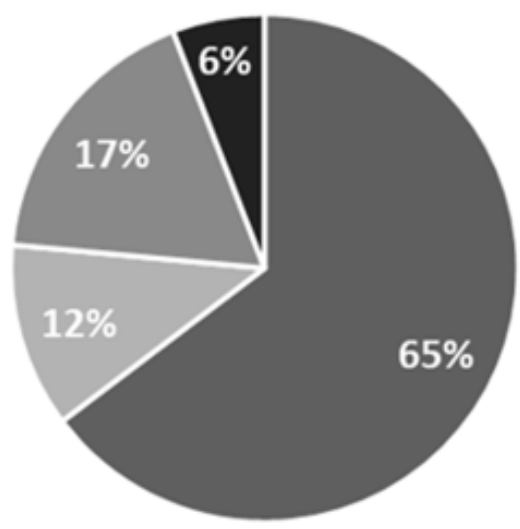

" Male $\|$ Female $\quad$ Both sexes $\quad$ No indicated

Figure 2: Percentage of studies using male animals, female animals, animals from both sexes or animals whose sex is not indicated.

Sex-specific differences in the microbiota can influence chronic disease [67], as is the case with the changes in the gut microbiome observed in patients with AD [68]. Female mice have higher Gammaproteobacteria abundance than males regardless of age, genotype, or diet. With aging, there are broad changes at the phylum level, with higher Proteobacteria and lower Firmicutes prevalence in females compared to males. Furthermore, aged females have higher Bacteroides and lower Faecalibaculum prevalence compared to males. Specific changes in the gut microbiota have been linked to $A \beta$ levels, with greater effects in females than in males. Bacteroides colonization increases $A \beta$ deposition, which ca be a mechanism whereby the gut affects $\mathrm{AD}$ pathogenesis. One option to treat $\mathrm{AD}$ is based on a simple method of calorie restriction because it induces changes in the microbiome. Thus, this highlights the importance of the sex-specific microbial interactions observed in $\mathrm{AD}$, suggesting that separate therapies may be needed for males and females [36].

One study demonstrated the potential sex dependence of the effect of the microbiome on $\mathrm{AD}$, showing the importance of the interactive effects of the apolipoprotein epsilon4 allele (APOE4), and sex on the relationship between gut microbiome and AD. APOE4 is the greatest genetic risk factor for AD [48]. Another study used a prebiotic such as inulin to effectively alter the gut microbiome in a preclinical mouse model of $\mathrm{AD}$, enhancing systemic metabolic functions and reducing the brain inflammation characteristic of early AD without showing any differences between the two sexes [40].

The microbiota of adult female mice has been reported to be similar to that of prepubescent female mice. However, postpubescent male mice show a changing microbial composition in their fecal content. This might result in a vulnerable ecosystem that is more open to being injured by toxic $A \beta$ peptides [37]. In general, it is clear that $\mathrm{AD}$ has a higher incidence in women than in men, with research on therapies for this disease still showing a sex bias against women. Sex is a fundamental biological variable that has to be considered in preclinical studies. The underrepresentation of female cells and animals in preclinical research has resulted in a poorer understanding of the biological, physiological, and pathophysiological mechanisms in females compared to males. Without data from females, it is impossible to determine whether the results obtained from male cells and animals also apply to female cells and animals. Basic science is necessary for the of new therapeutics. The development of animal models of diseases using only one sex makes it difficult to translate preclinical findings to the treatment of men and women [21]. There is a clear policy about 
the introduction of sex as biological variable in preclinical studies, and in this sense, the role of journals is crucial in advancing the quality and transparency of the data reported in research. However, progress in incorporating the topic of sex is very slow, especially in some areas such as neuroscience. A significant effort is needed from all those involved and especially from researches themselves.

The Sex and Gender Equity in Research (SAGER) guidelines are a comprehensive procedure for reporting gender information in study design, data analyses, results and data interpretation. The SAGER guidelines are designed for authors preparing their manuscripts, and also for editors who assess sex-related differences into all manuscripts submitted to for publication in their journals [69]. In conclusion, this review of sex bias in a specific case such as $\mathrm{AD}$ research shows that there is still a gap in preclinical studies despite recommendations and knowledge obtained about $\mathrm{AD}$ and its treatment will not be enough as long as this sex bias continues at the detriment of women. But unfortunately, this is not the only case and there are still many preclinical studies of different human diseases and treatment using only laboratory animals of one sex.

\section{Conclusion}

In conclusion, this review of sex bias in a specific case such as $\mathrm{AD}$ research shows that there is still a gap in preclinical studies despite recommendations. The knowledge about AD and its treatment will not be enough as long as this sex bias continues at the detriment of women. But unfortunately, this is not the only case and there are still many preclinical studies of different human diseases and their treatments using only laboratory animals of one sex and this need a change.

\section{Conflicts of Interest}

There are no conflicts of interest

\section{Acknowledgment}

Not applicable' for that section.

\section{References}

1. Scheltens P, Blennow K, Breteler MM, de Strooper B, Frisoni GB et al. (2016) Alzheimer's disease. Lancet 388: 505-517.

2. Knopman DS, Jack CR, Lundt ES, Weigand SD, Vemuri P, et al. (2016) Evolution of neurodegeneration-imaging biomarkers from clinically normal to dementia in the Alzheimer disease spectrum. Neurobiol Aging 46: $32-42$.

3. Ricciarelli R, Fedele E (2017) The Amyloid Cascade Hypothesis in Alzheimer's Disease: It's Time to Change Our Mind. Curr Neuropharmacol 15(6): 926-935.

4. Eckburg PB, Bik EM, Bernstein CN, Purdom E, Dethlefsen L, et al. (2005) Diversity of the human intestinal microbial flora. Science. 308: 16351638.

5. Cox LM, Weiner HL (2018) Microbiota Signaling Pathways that Influence Neurologic Disease. Neurotherapeutics 15: 135-145.

6. Fox M, Knorr DA, Haptonstall KM (2019) Alzheimer's disease and symbiotic microbiota: an evolutionary medicine perspective. Ann NY Acad 1449(1): 3-24.
7. O'Toole PW, Jeffery IB (2018) Microbiome-health interactions in older people. Cell Mol Life Sci CMLS 75: 119-128.

8. Hao X, Pan J, Gao X, Zhang S, Li Y (2021) Gut microbiota on gender bias in autism spectrum disorder. Rev 69 Neurosci 32: 69-77.

9. Liu F, Li J, Wu F, et al. Peng Q, Zhou H (2019) Altered composition and function of intestinal microbiota in autism spectrum disorders: a systematic review. Transl Psychiatry 9: 43.

10. Rieder R, Wisniewski PJ, Alderman BL, Campbell SC (2017) Microbes and mental health: A review. Brain Behave. Immunity 66: 9-17.

11. Hill JM, Lukiw WJ (2015) Microbial-generated amyloids and Alzheimer's disease (AD). Front. Aging Neurosci 7: 9.

12. Ambrosini YM, Borcherding D, Kanthasamy A, Kim HJ, Willette AA et al., (2019) The Gut-Brain Axis in Neurodegenerative Diseases and Relevance of the Canine Model: A Review. Front Aging Neurosci 11: 130.

13. Giau VV, Wu SY, Jamerlan A, An SSA, Kim SY, et al. (2018) Gut Microbiota and Their Neuroinflammatory Implications in Alzheimer's Disease. Nutrients 10(11): 1765

14. Liu S, Gao J, Zhu M, Liu K, Zhang HL (2020) Gut Microbiota and Dysbiosis in Alzheimer's Disease: Implications for Pathogenesis and Treatment. Mol Neurobiol 57(12): 5026-5043.

15. Moschetti K, Cummings PL, Sorvillo F, Kuo T (2012) Burden of Alzheimer's disease-related mortality in the United States, 1999-2008. J Am Geriatr Soc 60: 1509-1514.

16. Sanz A, Hiona A, Kujoth GC, Seo AY, Hofer T, et al. (2007) Evaluation of sex differences on mitochondrial bioenergetics and apoptosis in mice. Exp Gerontol 42: 173-182.

17. Nebel RA, Aggarwal NT, Barnes LL. Gallagher A, Goldstein JM, et al. (2018) Understanding the impact of sex and gender in Alzheimer's disease: A call to action. Alzheimers Dement 14(9): 1171-1183.

18. National Institutes of Health Revitalization Act of 1993. Subtitle Bclinical research equity regarding women and minorities. https://orwh. od.nih.gov/sites/orwh/files/docs/NIH-Revitalization-Act-1993.pdf. [accessed 07/05/2021].

19. Murthy VH, Krumholz HM, Gross CP (2004) Participation in cancer clinical trials: race-, sex-, and age-based disparities. JAMA 291(22): 2720-2726.

20. Kalliainen LK, Wisecarver I, Cummings A, Stone J (2018) Sex bias in hand surgery research. J Hand Surg 43(11): 1026-1029.

21. Sandberg K, Umans JG (2015) Georgetown Consensus Conference Work Group. Recommendations concerning the new U.S. National Institutes of Health initiative to balance the sex of cells and animals in preclinical research. FASEB J 29(5): 1646-52.

22. Whitley H, Lindsey W (2009) Sex-based differences in drug activity. Am Fam Physician 80(11): 1254-1258.

23. Feldman S, Ammar W, Lo K, Trepman E, van Zuylen M, et al. (2019) Quantifying Sex Bias in Clinical Studies at Scale With Automated Data Extraction. JAMA Netw Open 2(7): e196700.

24. Drummond E, Wisniewski T (2017) Alzheimer's disease: experimental models and reality. Acta Neuropathol 133(2): 155-175.

25. Leon WC, Canneva F, Partridge V, Allard S, Ferretti MT, et al. (2010) A novel transgenic rat model with a full Alzheimer's-like amyloid pathology displays pre-plaque intracellular amyloid-beta-associated cognitive impairment. J Alzheimers Dis 20: 113-126.

26. Kim MS, Kim Y, Choi H, Kim W, Park S, et al. (2020) Transfer of a healthy microbiota reduces amyloid and tau pathology in an Alzheimer's disease animal model. Gut 69(2): 283-294.

27. Korevaar DA, Hooft L, ter Riet G (2011) Systematic reviews and metaanalyses of preclinical studies: publication bias in laboratory animal experiments. Lab Anim 45: 225-230. 
28. Leenaars C, Tsaioun K, Stafleu F, Rooney K, Meijboom F, et al. (2020) Reviewing the animal literature: how to describe and choose between different types of literature reviews. Lab Anim 55:129-141.

29. Abraham D, Feher J, Scuderi GL, Szabo D, Dobolyi A, et al. (2019) Exercise and probiotics attenuate the development of Alzheimer's disease in transgenic mice: Role of microbiome. Exp Gerontol 115: 122-131.

30. Athari Nik Azm, S, Djazayeri A, Safa M, Azami K, Ahmadvand B, et al (2018) Lactobacilli and bifidobacteria ameliorate memory and learning deficits and oxidative stress in $\beta$-amyloid (1-42) injected rats. Appl Physiol Nutr Metab 43(7): 718-726.

31. Bäuerl C, Collado MC, Diaz Cuevas A, Viña J, Pérez Martínez G (2018) Shifts in gut microbiota composition in an APP/PSS1 transgenic mouse model of Alzheimer's disease during lifespan. Lett Appl Microbiol 66(6): 464-471.

32. Bonfili L, Cecarini V, Berardi S, Scarpona S, Suchodolski JS, et al. (2017) Microbiota modulation counteracts Alzheimer's disease progression influencing neuronal proteolysis and gut hormones plasma levels. Sci Rep 7(1): 2426.

33. Bonfili L, Cecarini V, Cuccioloni M, Angeletti M, Berardi S, et al. (2018) SLAB51 Probiotic Formulation Activates SIRT1 Pathway Promoting Antioxidant and Neuroprotective Effects in an AD Mouse Model. Mol Neurobiol 55(10): 7987-8000.

34. Bonfili L, Cecarini V, Gogoi O, Berardi S, Scarpona S, et al. (2020) Gut microbiota manipulation through probiotics oral administration restores glucose homeostasis in a mouse model of Alzheimer's disease. Neurobiol Aging 87: 35-43.

35. Brandscheid C, Schuck F, Reinhardt S, Schäfer KH, Pietrzik CU, et al. (2017) Altered Gut Microbiome Composition and Tryptic Activity of the 5xFAD Alzheimer's Mouse Model. J Alzheimers Dis 56(2): 775-788.

36. Cox LM, Schafer MJ, Sohn J, Vincentini J, Weiner HL, et al. (2019) Calorie restriction slows age-related microbiota changes in an Alzheimer's disease model in female mice. Sci Rep 9(1): 17904

37. Cui B, Su D, Li W, She X, She X, et al. (2018) Effects of chronic noise exposure on the microbiome-gut-brain axis in senescence-accelerated prone mice: implications for Alzheimer's disease. J Neuroinflammation 15(1): 190.

38. Fujii Y, Nguyen TTT, Fujimura Y, Kameya N, Nakamura S, et al. (2019) Fecal metabolite of a gnotobiotic mouse transplanted with gut microbiota from a patient with Alzheimer's disease. Biosci Biotechnol Biochem 83(11): 2144-2152.

39. Gao Q, Wang Y, Wang X, Fu S, Zhang X, et al. (2019) Decreased levels of circulating trimethylamine $\mathrm{N}$-oxide alleviate cognitive and pathological deterioration in transgenic mice: a potential therapeutic approach for Alzheimer's disease. Aging (Albany NY) 11(19): 8642-8663.

40. Hoffman JD, Yanckello LM, Chlipala G, Hammond TC, McCulloch SD, et al. (2019) Dietary inulin alters the gut microbiome, enhances systemic metabolism and reduces neuroinflammation in an APOE4 mouse model. PLoS One 14(8): e0221828.

41. Kaur H, Nagamoto-Combs K, Golovko S, Golovko MY, Klug MG, et al. (2020) Probiotics ameliorate intestinal pathophysiology in a mouse model of Alzheimer's disease. Neurobiol Aging 92: 114-134.

42. Kim DS, Ko BS, Ryuk JA, Park S (2020) Tetragonia tetragonioides Protected against Memory Dysfunction by Elevating Hippocampal Amyloid- $\beta$ Deposition through Potentiating Insulin Signaling and Altering Gut Microbiome Composition. Int J Mol Sci 21(8): 2900.

43. Li Z, Zhu H, Guo Y, Du X, Qin C (2020) Gut microbiota regulates cognitive deficits and amyloid deposition in a model of Alzheimer's disease. J Neurochem 155(4): 448-461.

44. Liu Y, Du T, Zhang W, Lu W, Peng Z, et al. (2019) Modified Huang-LianJie-Du Decoction Ameliorates $A \beta$ Synaptotoxicity in a Murine Model of Alzheimer's Disease. Oxid Med Cell Longev: 8340192.

45. Lv M, Yang S, Cai L, Qin LQ, Li BY et al. (2018) Effects of Quercetin Intervention on Cognition Function in APP/PS1 Mice was Affected by Vitamin D Status. Mol Nutr Food Res 62(24): e1800621.
46. Minter MR, Zhang C, Leone V, Ringus DL, Zhang X, et al. (2016) Antibiotic-induced perturbations in gut microbial diversity influences neuro-inflammation and amyloidosis in a murine model of Alzheimer's disease. Sci Rep 6: 30028.

47. Minter MR, Hinterleitner R, Meisel M, Zhang C, Leone V, et al. (2017) Antibiotic-induced perturbations in microbial diversity during postnatal development alters amyloid pathology in an aged APPSWE/ PS1 $\Delta$ E9 murine model of Alzheimer's disease. Sci Rep 7: 10411.

48. Maldonado Weng J, Parikh I, Naqib A, York J, Green SJ, et al. (2019) Synergistic effects of APOE and sex on the gut microbiome of young EFAD transgenic mice. Mol Neurodegener 14(1): 47.

49. Mohammadi G, Dargahi L, Peymani A, Mirzanejad Y, Alizadeh SA et al. (2019) The Effects of Probiotic Formulation Pretreatment (Lactobacillus helveticus R0052 and Bifidobacterium longum R0175) on a Lipopolysaccharide Rat Model. J Am Coll Nutr 38(3): 209-217.

50. Peng W, Yi P, Yang J, Xu P, Wang Y, et al. (2018) Association of gut microbiota composition and function with a senescence-accelerated mouse model of Alzheimer's Disease using 16S rRNA gene and metagenomic sequencing analysis. Aging (Albany NY) 10(12): 4054 4065 .

51. Rezaei Asl Z, Sepehri G, Salami M (2019) Probiotic treatment improves the impaired spatial cognitive performance and restores synaptic in an animal model of Alzheimer's disease. Behav Brain Res 376: 112183.

52. Shen L, Liu L, Ji HF (2018) Alzheimer's Disease Histological and Behavioral Manifestations in Transgenic Mice Correlate with Specific Gut Microbiome State. J Alzheimers Dis 56(1): 385-390.

53. Shen L, Liu L, Li XY, Ji HF (2019) Regulation of gut microbiota in Alzheimer's disease mice by silibinin and silymarin and their pharmacological implications. Appl Microbiol Biotechnol 103(17): 7141-7149.

54. Sun J, Xu J, Ling Y, Wang F, Gong T, et al. (2019) Fecal microbiota transplantation alleviated Alzheimer's disease-like pathogenesis in APP/PS1 transgenic mice. Transl Psychiatry 9(1): 189.

55. Sun BL, Li WW, Wang J, Xu YL, Sun HL, et al. (2019) Gut Microbiota Alteration and Its Time Course in a Tauopathy Mouse Model. J Alzheimers Dis 70(2): 399-412.

56. Sun J, Liu S, Ling Z, Wang F, Ling Y, et al. (2019) Fructooligosaccharides Ameliorating Cognitive Deficits and Neurodegeneration in APP/PS1 Transgenic Mice through Modulating Gut Microbiota. J Agric Food Chem 67(10): 3006-3017.

57. Syeda T, Sanchez-Tapia M, Pinedo-Vargas L, Granados O, Cuervo-Zanatta D, et al. (2018) Bioactive Food Abates Metabolic and Synaptic Alterations by Modulation of Gut Microbiota in a Mouse Model of Alzheimer's Disease. J Alzheimer Dis 66(4): 1657-1682.

58. Wang J, Ye F, Cheng X, Zhang X, Liu F, et al. (2016) The Effects of LWAFC on Intestinal Microbiome in Senescence-Accelerated Mouse Prone 8 Strain, a Mouse Model of Alzheimer's Disease. J Alzheimers Dis 53(3): 907-919.

59. Wang X, Sun G, Feng T, Zhang J, HuangX, etal. (2019) Sodium oligomannate therapeutically remodels gut microbiota and suppresses gut bacterial amino acids-shaped neuroinflammation to inhibit Alzheimer's disease progression. Cell Res 29(10): 787-803.

60. Wang J, Lei X, Xie Z, Zhang X, Cheng X, et al. (2019) CA-30, an oligosaccharide fraction derived from Liuwei Dihuang decoction, ameliorates cognitive deterioration via the intestinal microbiome in the senescence-accelerated mouse prone 8 strain. Aging (Albany NY) 11(11): 3463-3486

61. Xin Y, Diling C, Jian Y, Ting L, Guoyan H, et al. (2018) Effects of Oligosaccharides from Morinda officinalis on Gut Microbiota and Metabolome of APP/PS1 Transgenic Mice. Front Neurol 9: 412

62. Zhang L, Wang Y, Xiayu X, Shi C, Chen W, et al. (2017) Altered Gut Microbiota in a Mouse Model of Alzheimer's Disease. J Alzheimers Dis 60(4): 1241-1257.

63. Beery AK, Zucker I (2011) Sex bias in neuroscience and biomedical research. Neurosci Biobehav Rev 35(3): 565-572. 
64. Will TR, Proaño SB, Thomas AM, Kunz LM, Thompson KC, et al. (2017) Problems and Progress regarding Sex Bias and Omission in Neuroscience Research. eNeuro 4(6): ENEURO.0278-17.

65. Manocha GD, Floden AM, Miller NM, Smith AJ, Nagamoto-Combs K, et al. (2019) Temporal progression of Alzheimer's disease in brains and intestines of transgenic mice. Neurobiol Aging 81: 166-176.

66. Dodiya HB, Kuntz T, Shaik SM, Baufeld C, Leibowitz J, et al. (2019) Sex-specific effects of microbiome perturbations on cerebral Abeta amyloidosis and microglia phenotypes. J Exp Med 216: 1542-1560.
67. Markle JG, Frank DN, Mortin-Toth S, Robertson CE, Feazel LM, et al. (2013) Sex differences in the gut microbiome drive hormone-dependent regulation of autoimmunity. Science 339(6123): 1084-1088.

68. Zhuang ZQ, Shen LL, Li WW, Fu X, Zeng F, et al. (2018) Gut Microbiota is Altered in Patients with Alzheimer's Disease. J Alzheimers Dis 63(4): 1337-1346.

69. Heidari S, Babor TF, De Castro P, ort S, Curno M (2016) Sex and Gender Equity in Research: rationale for the SAGER guidelines and recommended use. Res Integr Peer Rev 1: 2. 